\title{
Erratum to: Hepatocellular Carcinomas within the Milan Criteria: Predictors of Histologic Necrosis after Drug-eluting Beads Transarterial Chemoembolization
}

\author{
Bruno C. Odisio - Francisco Galastri - Rony Avritscher - Breno B. Afonso • \\ Vanderlei Segatelli • Guilherme E. G. Felga • Paolo R. O. Salvalaggio • \\ Joe Ensor $\cdot$ Michael J. Wallace $\cdot$ Felipe Nasser
}

Published online: 19 November 2013

(C) Springer Science+Business Media New York and the Cardiovascular and Interventional Radiological Society of Europe (CIRSE) 2013

\section{Erratum to: Cardiovasc Intervent Radiol \\ DOI 10.1007/s00270-013-0759-z}

The publisher regrets causing the following errors in the article:

\section{Results}

Table 2:

Table Title: "Imaging pathology" should be replaced by "Imaging-pathology".

Total mRECIST: CR, 18; PR, 7; SD, 2.

The online version of the original article can be found under doi:10.1007/s00270-013-0759-z.

\section{B. C. Odisio $(\square) \cdot$ R. Avritscher · M. J. Wallace}

Department of Interventional Radiology, Division of Diagnostic Imaging, The University of Texas MD Anderson Cancer Center, 1400 Pressler Street, Unit 1471, Houston, TX 77030-3722, USA e-mail: bcodisio@mdanderson.org

\section{R. Avritscher}

e-mail: ravritscher@mdanderson.org

M. J. Wallace

e-mail: mwallace@mdanderson.org

F. Galastri - B. B. Afonso · F. Nasser

Division of Interventional Radiology, Hospital Israelita Albert

Einstein, Av. Albert Einstein, 627, São Paulo,

SP 05652-900, Brazil

e-mail: leogalastri@hotmail.com

B. B. Afonso

e-mail: Breno@einstein.br

F. Nasser

e-mail: felipenasser@einstein.br
Footnote: all patients were deemed within Milan criteria at the time of DEB-TACE procedure and study enrolment. Lesion diameters for No. 10 and 16 reflect values of final radiology report issued after the first DEB-TACE procedure, which differed from the preliminary radiology report and the interventional radiologist's assessment.

\section{Discussion}

First paragraph, second line, "small $(\leq 3 \mathrm{~cm})$ HCCs" should be replaced for "HCCs measuring $\leq 5 \mathrm{~cm}$ ".

\author{
V. Segatelli \\ Division of Pathology, Hospital Israelita Albert Einstein, \\ Av. Albert Einstein, 627, São Paulo, SP 05652-900, Brazil \\ e-mail: vanderlei.segatelli@einstein.br \\ G. E. G. Felga · P. R. O. Salvalaggio \\ Division of Liver Transplantation, Hospital Israelita Albert \\ Einstein, Av. Albert Einstein, 627, São Paulo, \\ SP 05652-900, Brazil \\ e-mail: guilherme.felga@einstein.br \\ P. R. O. Salvalaggio \\ e-mail: paolo.salvalaggio@einstein.br \\ J. Ensor \\ Department of Biostatistics, The University of Texas MD \\ Anderson Cancer Center, 1400 Pressler Street, Unit 1471, \\ Houston, TX 77030-3722, USA \\ e-mail: joensor@mdanderson.org
}

